\title{
Comparison of Currents on Upper and Lower Sides of Patch Conductor of Microstrip Antenna
}

\author{
Takafumi FUJIMOTO*, Kazumasa TANAKA, Mitsuo TAGUCHI \\ Dept. of Electrical \& Electronic Eng., Nagasaki University \\ 1-14 Bunkyo-machi, Nagasaki-shi, 852-8521, Japan \\ E-mail: takafumi@net.nagasaki-u.ac.jp
}

\section{Introduction}

In the analysis of a microstrip antenna (MSA) by the method of moment in the spectral domain $(\mathrm{SD}-\mathrm{MoM})[1]$, the patch conductor is assumed to be infinitely thin, and the total electric currents on the upper and lower sides of the patch conductor are derived.

In this paper, the electric currents on the upper and lower sides of the patch conductor are derived by SD-MoM with consideration of the thickness of the patch conductor. Integral equations are derived from the boundary condition that the total tangential electric field vanishes on the upper and lower sides of patch conductor. The total electric field is derived by using Green's functions in the spectral domain produced by the electric dipoles on the upper and lower sides of the patch conductor.

In order to clarify the effects of the currents on the upper and lower sides of the patch to the antenna, the input impedances due to those currents are calculated.

\section{Theory}

Fig. 1 shows the geometry of a circular MSA and its coordinate system. The radius and thickness of the circular patch conductor are $a_{0}$ and $\delta_{z}$, respectively. The relative dielectric constant and thickness of the dielectric substrate are $\varepsilon_{r}$ and $h$, respectively. The antenna is excited at $r=d_{0}, \phi=0^{\circ}$ by a coaxial feeder through the dielectric substrate.

The electric currents on the upper and lower sides of the patch conductor are denoted by $\boldsymbol{J}^{U}$ and $\boldsymbol{J}^{L}$, respectively. Fig. 2 shows an analytical model. The patch conductor is expressed by two infinitely thin conducting sheets. $\boldsymbol{J}^{U}$ and $\boldsymbol{J}^{L}$ are assumed to flow on the upper and lower sheets. The currents on the patch conductor follow closely the behavior of the corresponding eigenmode within the cavity bounded above and below by the conducting plates and on the side by the admittance wall[2]. Therefore, $\boldsymbol{J}^{U}$ and $\boldsymbol{J}^{L}$ are expressed as

$$
\begin{aligned}
\boldsymbol{J}^{i} & =J_{r}^{i}(r, \phi) \boldsymbol{i}_{r}+J_{\phi}^{i}(r, \phi) \boldsymbol{i}_{\phi} \\
J_{r}^{i} & =\sum_{m=0}^{M} \sum_{n=0}^{N} A_{m n}^{i} U_{m}\left(\frac{r}{a_{0}}\right) \sqrt{1-\left(\frac{r}{a_{0}}\right)^{2}} \cos (n \phi) \\
J_{\phi}^{i} & =\sum_{m=0}^{M} \sum_{n=0}^{N} B_{m n}^{i} T_{m}\left(\frac{r}{a_{0}}\right) \frac{1}{\sqrt{1-\left(\frac{r}{a_{0}}\right)^{2}}} \sin (n \phi) \quad, \quad i=U \text { or } L .
\end{aligned}
$$


In eqns (1) the polynomials under the square root are included to fulfill the edge condition requirements. $T_{n}\left(r / a_{0}\right)$ and $U_{n}\left(r / a_{0}\right)$ are Chebyshev polynomials of the first and second kind, respectively. $\left\{A_{m n}^{i}\right\}$ and $\left\{B_{m n}^{i}\right\}$ are unknown coefficients.

The electric fields on the upper and lower sides of the patch conductor produced by $\boldsymbol{J}^{i}$ are denoted by $\boldsymbol{E}^{U}\left(\boldsymbol{J}^{i}\right)$ and $\boldsymbol{E}^{L}\left(\boldsymbol{J}^{i}\right)$, respectively. The excitation fields due to the feed current $\boldsymbol{J}^{e}$ given by the delta function are denoted by $\boldsymbol{E}_{e}^{U}\left(\boldsymbol{J}^{e}\right)$ and $\boldsymbol{E}_{e}^{L}\left(\boldsymbol{J}^{e}\right)$. The boundary conditions on the upper and lower sides of the patch conductor are expressed as

$$
\begin{aligned}
\left\{\boldsymbol{E}^{U}\left(\boldsymbol{J}^{U}\right)+\boldsymbol{E}^{U}\left(\boldsymbol{J}^{L}\right)+\boldsymbol{E}_{e}^{U}\left(\boldsymbol{J}^{e}\right)\right\} \times \boldsymbol{i}_{z} & =\mathbf{0}: z=h+\delta_{z} \\
\left\{\boldsymbol{E}^{L}\left(\boldsymbol{J}^{U}\right)+\boldsymbol{E}^{L}\left(\boldsymbol{J}^{L}\right)+\boldsymbol{E}_{e}^{L}\left(\boldsymbol{J}^{e}\right)\right\} \times\left(-\boldsymbol{i}_{z}\right) & =\mathbf{0}: z=h .
\end{aligned}
$$

In the derivation of $\boldsymbol{E}^{j}\left(\boldsymbol{J}^{i}\right)(j=U, L, i=U, L)$, the local coordinate system $(X, Y, Z)$ with the origin located at the point $\left(r^{\prime}, \phi^{\prime}, 0\right)$ in the cylindrical coordinate system is applied for the circular MSA. Fig. 3 shows the local coordinate system. The positive $X$ direction is defined by the tangential $\phi^{\prime}$ direction. $\boldsymbol{E}^{j}\left(\boldsymbol{J}^{i}\right)$ is expressed by using the vector potential $\boldsymbol{A}^{j}\left(\boldsymbol{J}^{i}\right)$ and the scalar potential $\phi_{e}^{j}\left(\boldsymbol{J}^{i}\right)$;

$$
\begin{aligned}
\boldsymbol{E}^{j}\left(\boldsymbol{J}^{i}\right) & =-j \omega \boldsymbol{A}^{j}\left(\boldsymbol{J}^{i}\right)-\nabla \phi_{e}^{j}\left(\boldsymbol{J}^{i}\right) \\
\boldsymbol{A}^{j}\left(\boldsymbol{J}^{i}\right) & =\int_{\text {patch }}\left\{\boldsymbol{i}_{X} G_{A}^{X X}(j \mid i) J_{\phi}^{i}-\boldsymbol{i}_{Y} G_{A}^{Y Y}(j \mid i) J_{r}^{i}\right\} d S^{\prime} \\
\phi_{e}^{j}\left(\boldsymbol{J}^{i}\right) & =-\frac{1}{j \omega} \int_{\text {patch }} G_{U}(j \mid i)\left(\nabla^{\prime} \cdot \boldsymbol{J}^{i}\right) d S^{\prime}
\end{aligned}
$$

where $G_{A}^{S T}$ is $S$ component of Green's function for the vector potential due to a $T$ - directed electric dipole and $G_{U}$ is Green's function for the scalar potential. $\nabla$ and $\nabla^{\prime}$ are the derivative operators at the observation and source points. Substituting eqns. (4)-(6) into eqns. (2) and (3), the integral equations are obtained. By applying the method of moment to the integral equations, $\left\{A_{m n}^{i}\right\}$ and $\left\{B_{m n}^{i}\right\}$ are determined. When $\delta_{z}=0$, the integral equations obtained from eqns. (2) and (3) are equal to that of reference [1], where the unknowns are the total currents on the upper and lower sides of the patch conductor.

Green's functions in the spectral domain are obtained by applying the solutions of the wave equations in the spectral domain to the boundary conditions at the interfaces between the free space, the dielectric and the ground plane and the radiation condition. Green's functions in the spatial domain are derived by applying the inverse Fourier transform to Green's functions in the spectral domain [1].

\section{Results and Discussion}

Figs. 4 and 5 show the calculated $J_{r}^{U}, J_{r}^{L}$ and $J_{\phi}^{U}, J_{\phi}^{L}$ at the resonant frequency $(6.22 \mathrm{GHz})$, respectively. The intensity of $J_{r}^{U}$ is bigger than $J_{r}^{L}$. However, the intensity of $J_{\phi}^{U}$ is smaller than $J_{\phi}^{L}$. The phase of the $J_{r}^{U}$ is nearly equal to that of $J_{r}^{L}$. Although the phase of $J_{\phi}^{L}$ is constant, $J_{\phi}^{U}$ has the phase difference $180^{\circ}$ in the $r$ direction.

Fig. 6 shows the calculated input impedances. $\boldsymbol{J}^{U}$ doesn't contribute to the input impedance. This is due to the phase difference of $J_{\phi}^{U}$ in the $r$ direction. 


\section{Conclusion}

The electric currents on the upper and lower sides of the patch have been calculated by SD-MoM. Green's functions produced by the electric dipoles on the upper and lower sides of the patch are derived by the boundary conditions at the interfaces between the free space, the dielectric and the ground plane and the radiation condition. In order to clarify the effects of those currents to the antenna, the input impedances by those currents have been compared. The input impedance of the MSA depends on the current on the lower side of the patch conductor.

\section{Acknowledgment}

This research was supported in part by Grants-in-Aid for Scientific Research 13750361 from Japan Society for the Promotion of Science.

\section{References}

[1] J.R. Mosig, "Integral equation technique, " ed. T. Itoh, in Numerical techniques for microwave and millimeter-wave passive structures, pp.133-213, John Wiley \& Sons, New York, 1989.

[2] T. Fujimoto, K. Tanaka and M. Taguchi, "Wall admittance of a circular microstrip antenna," IEICE Trans. Commun.,, vol. E82-B, no. 5, pp.760767, May 1999.

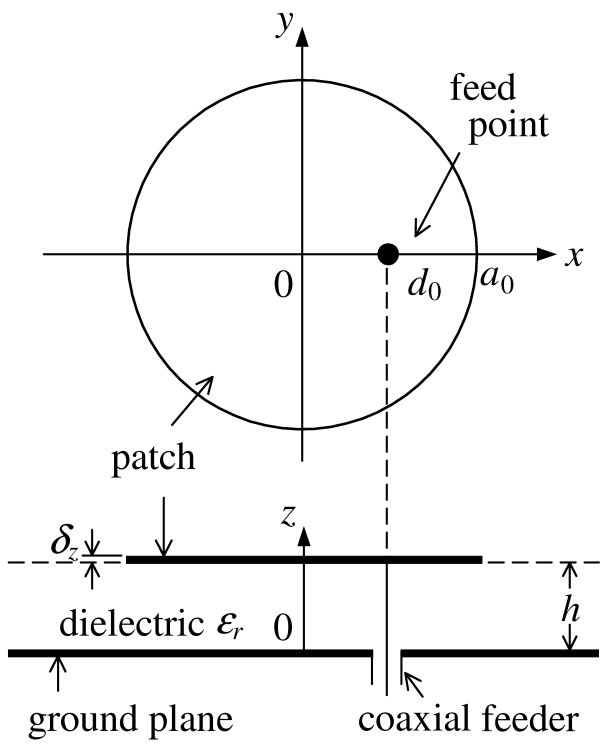

Fig. 1 Circular MSA

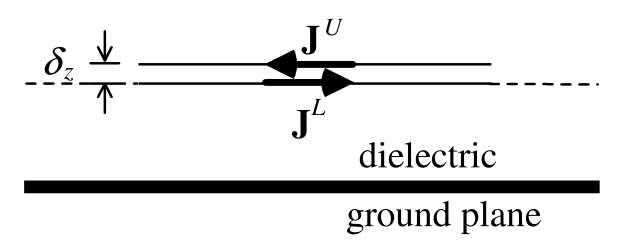

Fig. 2 Analytical model (cross section)

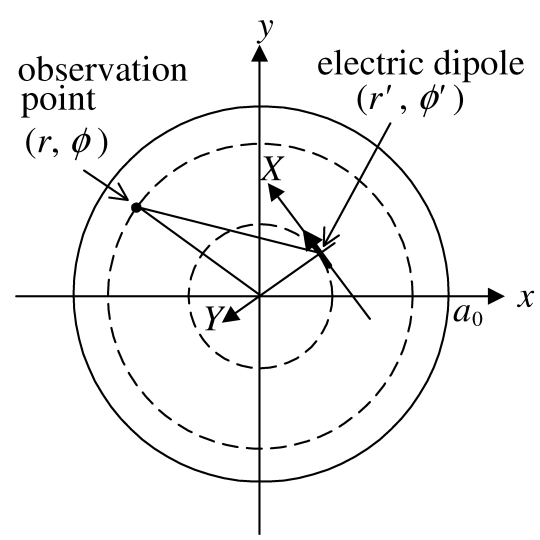

Fig. 3 Local coordinate system 


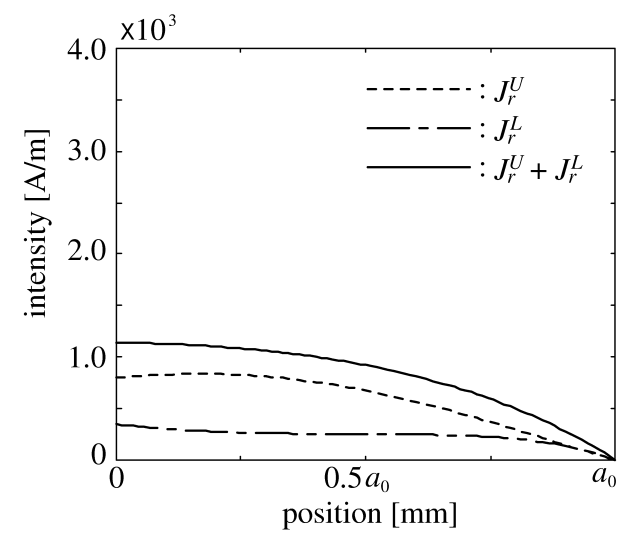

(a) Intensity

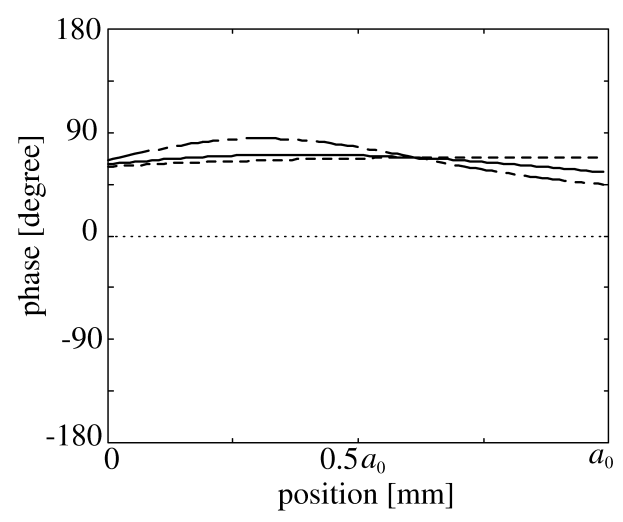

(b) Phase

Fig. $4 r$ component of electric currents $\left(\phi=0^{\circ}\right)$

( $a_{0}=9.06 \mathrm{~mm}, d_{0}=6.0 \mathrm{~mm}, h=0.764 \mathrm{~mm}, \varepsilon_{r}=2.15, \delta_{z}=0.018 \mathrm{~mm}, M=N=3$ )

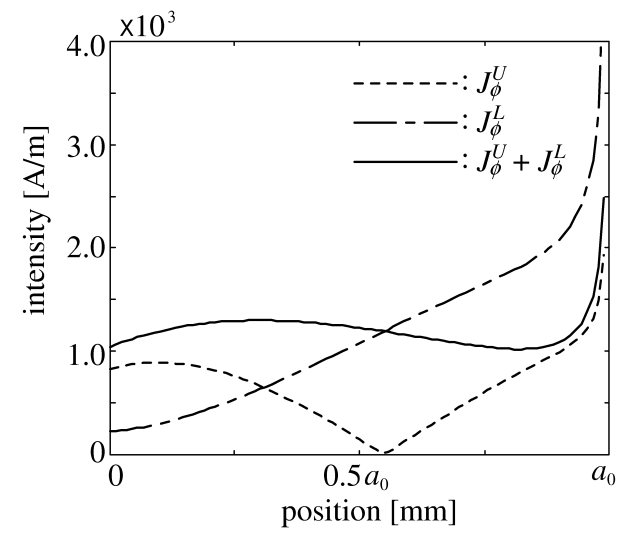

(a) Intensity

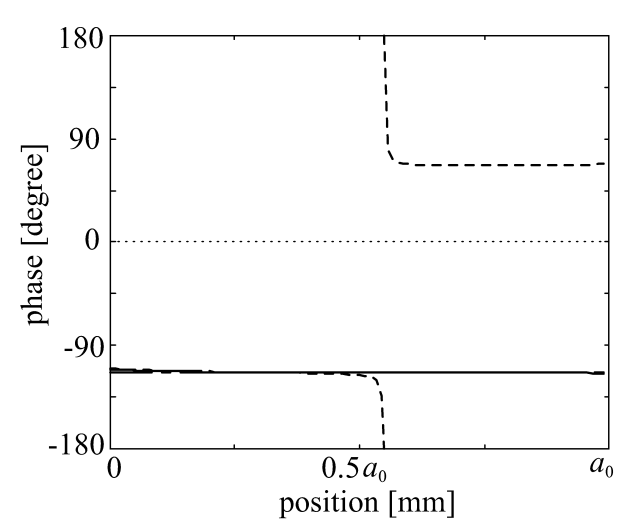

(b) Phase

Fig. $5 \phi$ component of electric currents $\left(\phi=90^{\circ}\right)$

( $a_{0}=9.06 \mathrm{~mm}, d_{0}=6.0 \mathrm{~mm}, h=0.764 \mathrm{~mm}, \varepsilon_{r}=2.15, \delta_{z}=0.018 \mathrm{~mm}, M=N=3$ )

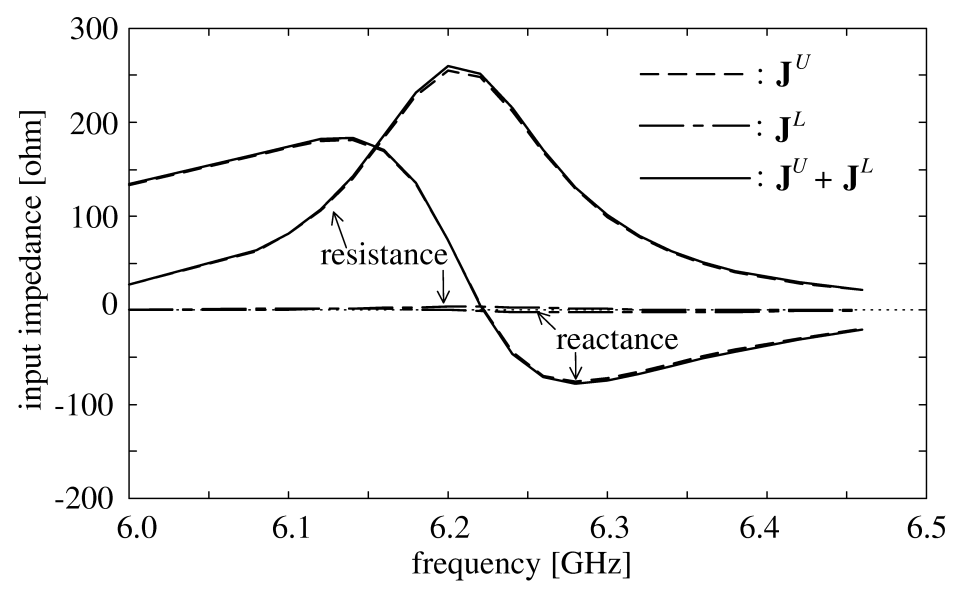

Fig. 6 Input impedances

$\left(a_{0}=9.06 \mathrm{~mm}, d_{0}=6.0 \mathrm{~mm}, h=0.764 \mathrm{~mm}, \varepsilon_{r}=2.15, \delta_{z}=0.018 \mathrm{~mm}, M=N=3\right)$ 